\title{
Modeling Melting in Binary Systems
}

\author{
Leonid D. Son \\ Ural Pedagogical University, Ekaterinburg 620219, Russia \\ German M. Rusakov, \\ Ural Polytechnical Institute, Ekaterinburg 620002, Russia \\ and \\ Alexander Z. Patashinski, \\ Department of Physics and Astronomy and Materials \\ Research Center, Northwestern University, Evanston IL \\ 60208, USA, and Budker Institute of Nuclear Physics, \\ Novosibirsk, Russia, \\ and \\ Mark A. Ratner, \\ Department of Chemistry and Materials Research Center, \\ Northwestern University, Evanston IL 60208, USA,
}

\begin{abstract}
A coarsened model for a binary system with limited miscibility of components is proposed; the system is described in terms of structural states in small parts of the material. The material is assumed to have two alternative types of crystalline local arrangements associated with two components of the alloy. Fluctuating characteristics of a cluster are the type and the space orientation of its crystalline arrangement. There are two different phase transitions in the model system, an orientation orderdisorder transition representing melting, and a phase transition between phases differing in concentration of components. Depending on the parameters characterizing the interaction in the system, this last transition may take place both in the crystalline and in the amorphous (molten) phase. A special approximation is used to study the thermodynamics of the system. The calculated phase diagram describes, at least qualitatively, the most important features of a binary system.
\end{abstract}




\section{Introduction.}

In binary systems, composition-temperature phase diagrams show the regions of phase stability, usually under constant pressure conditions [1]. Phase transitions in those systems include melting, which is a universal phenomenon in all crystalline materials, eventual phase transitions due to non-miscibility of components and, polymorphous phase transitions that may appear if there is a competition between different crystalline phases of the same material. The appearance of these latter transitions depends on the atomic scale interactions in the material. In isomorphous alloy systems (e.g. copper -nickel alloy), only a single type of crystal structure is observed. A more complicated picture appears in alloys with low miscibility of components. In those systems, clusters having different predominant components may differ in crystalline arrangements. A known example of an eutectic system is the copper-silver alloy, while that of a monotectic system is given by the copper-lead alloy. . Similarities of melting in different materials allow one to assume that the melting behavior depends on rather general characteristics (e.g. the particle size, first moments of the interaction potential) of the interparticle interactions, and that a coarsened statistical model operating with only a few energy parameters may reproduce the main features of those materials. In the present paper, we propose such a coarsened model in terms of structural states of the system small parts.

In liquids and solids, the interaction of constituent particles is strong and substantially limits the relative positions of particles. The resulting strong correlation in small volumes of the material is referred to as the local order in condensed systems. The term "local" refers to a small part of the material, whose size may be estimated from the correlation functions as including one or more coordination shells. We refer to this small part as a cluster. To account for the limitations imposed on relative positions of cluster particles by the local order, one describes the particle arrangements in terms of local order parameters. The concept of local order assumes a coarsening of description to a space scale larger than the interatomic distance. The resulting model is then a simplification that allows one to understand the global ordering in terms of the local order.

In simple liquids (e.g. noble gas liquids), radial correlation functions [2, 3] show some order in the first coordination shell, and a weaker correlation of particles positions in the second and higher correlation shells. The possibility to describe these liquids in terms of a definite local order is an open question. In liquids with stronger interaction of particles, and especially with covalent or/and hydrogen bonding, one expects the local order to be stronger, and closer to the local arrangement in a crystal. This will be referred to as a crystalline local arrangement.

In the current article, we consider a material in which the local order is maintained by a strong short range interaction, so that this local order does not significantly change even when the global order is lost by melting. In such a material, the loss of the global order during melting results from proliferation of topological defects, with the density of these defects small enough to allow 
recognition of the crystalline local structure. In the crystalline state, orientations of all clusters almost coincide, while in the liquid the orientations become uncorrelated above some finite distance. For a single component material or an isomorphous system with only one type of local order, a model of melting based on the above physical picture was studied earlier [4. A more general theory of melting that accounts for amorphous inherent structures is studied in a recent paper [5].

In a crystalline two-component alloy with limited miscibility of components, one deals at least with two types of local order corresponding to phases with the predominance of one component. In the materials considered, the shortrange interaction energetically favors, for each sort of atoms, the same sort of particles in the nearest neighborhood. The physical picture of the alloy is then a cluster-scale solution. Close to the phase separation caused by non-miscibility, the material is a solution of one type of clusters in the matrix of the other type of local structure [1. This suggestion determines the choice of cluster states in our model.

In what follows, we propose a model for an alloy based on the above physical picture. The meta-Hamiltonian of the model is written in terms of a local order parameter describing two possible predominant configurations in small elements of the material. The particle arrangement in such a small element is assumed to be of crystalline type, with orientations of these crystalline arrangements being the fluctuating characteristics. We study the phase transitions and the phase diagram in this model.

\section{The local state model of an alloy.}

We divide the material in equal small parts (clusters) of cubic shape. The centers of these clusters form a simple cubic lattice with sites having coordinates $r$. In accordance with the discussion in the previous section, we assume that the relative positions of cluster particles are, within the accuracy of small thermal displacements and possible packing defects, of a crystalline type. The crystalline local arrangement is anisotropic. One can characterize the space orientation of this anisotropy by introducing local crystalline axes. In the crystal state, the orientations of these axes are close to the corresponding orientations of some global axes characterizing the crystal on a macroscopic length scale. In a noncrystalline state, the orientation of local crystalline axes become, together with the type of the local crystalline anisotropy and the local composition, a fluctuating degree of freedom. In our model, we consider only this degrees of freedom. The universal contribution to thermodynamic properties coming from the small oscillations of atoms in the vicinity of an energy minimum configuration may be found, for a classical system, from a rather general consideration $\underline{6}$.

To simplify the modeling, we coarsen the orientation description in a way proposed in [4] 5]. Namely, we divide the orientation space of a cluster in orientation cells comprising only slightly differing orientations, and characterize the orientation state of a cluster by the number $i, i=\overline{1, N}$ of the orientation 
cell. The size of an orientation cell, and thus the number $N$ of orientation states, will be discussed later. We assign to a cluster situated at the point $r$ [7] an $N$-component vector $\sigma_{j}(r)$ by the condition that $\sigma_{j}(r)=1$ when the orientation state of the cluster is $j$, and $\sigma_{j}(r)=0$ otherwise. The global state of the material is characterized by the configuration $\left\{\sigma_{j}(r)\right\}$ and described by the field $\sigma_{j}(r)$ at all points $r$. Then, a statistical theory of the material may be formulated in terms of the field $\sigma_{j}(r)$ of the local order parameter.

One writes the probability $w\left\{\sigma_{i}(r)\right\}$ of a configuration $\left\{\sigma_{i}(r)\right\}$ as $w \sim$ $\exp \left[-H\left\{\sigma_{i}(r)\right\} / k_{B} T\right]$ where $k_{B}$ is the Boltzmann constant, and $H\left\{\sigma_{i}(r)\right\}$ is the meta-Hamiltonian of the system; below, we choose units in which $k_{B}=1$. The technique of meta-Hamiltonians (effective Hamiltonian, nonequilibrium thermodynamic potential) in the coarsened statistical description of condensed systems was developed in the theory of critical phenomena (see, e.g. 6, 8] ). To obtain $H\left\{\sigma_{i}(r)\right\}$, one has to take averages, with Gibbs measure, over all degrees of freedom except for those described by $\sigma_{i}(r)$. The meta-Hamiltonian $H\left\{\sigma_{i}(r)\right\}$ thus gives the free energy of the system in a global state characterized by given local states $\sigma_{i}(r)$ of each cluster. The the meta-Hamiltonian $H\left\{\sigma_{i}(r)\right\}$ depends, through coefficients defining the mathematical form of $H\left\{\sigma_{i}(r)\right\}$, on temperature $\mathrm{T}$, pressure $\mathrm{P}$, and other thermodynamic coordinates of the system. One may use these coefficients as new thermodynamic coordinates instead of $\mathrm{P}$ and/or $\mathrm{T}$. To be specific, we consider a system at a given pressure $\mathrm{P}$; the thermodynamic coordinates of the state are the temperature $\mathrm{T}$ and component concentrations. A change of the pressure will change, in general, the meta-Hamiltonian.

The form of the meta-Hamiltonian follows from rather general physical arguments 6 , 8. One expects $H$ to include independent contributions from single clusters (one-cluster part), each such contribution $\alpha_{i}$ depending only on the state $i$ of the corresponding cluster . The interaction of neighboring clusters is described by terms depending on states of more than one cluster. Here, we only consider two-cluster interactions. The reason is that the interacting potentials acting between particles have a range shorter then the cluster size, so the interaction is strong only for clusters sharing a border. Then, for a system in homogeneous external conditions, the general form of the meta-Hamiltonian is

$$
H\{\sigma\}=-\sum_{r, r^{\prime}} \sigma_{i}(r) M_{i j}\left(r-r^{\prime}\right) \sigma_{j}\left(r^{\prime}\right)-\sum_{r} \sigma_{i}(r) \alpha_{i}(r) .
$$

Here and below, the Einstein's summation rule is supposed for repeating indices. Let us define the field $\omega_{i}(r)$ as

$$
\begin{gathered}
<\sigma_{i}(r)>=\omega_{i}(r)=\frac{1}{Z} \sum_{\{\sigma\}} \sigma_{i}(r) \exp \left(-\frac{H\{\sigma\}}{T}\right), \\
Z=\sum_{\{\sigma\}} \exp \left(-\frac{H\{\sigma\}}{T}\right)
\end{gathered}
$$

the partition function $Z\left(\alpha_{i}(r), T\right)$ is a sum over all local states in all clusters. The quantity $\omega_{i}(r)$ defined by (2) is the probability that the cluster centered at 
point $r$ is in the state $i$, it yields the normalization condition

$$
\sum_{i=1}^{N} \omega_{i}(r)=1 .
$$

The partition function $Z$ is the generating functional for the field $\omega_{i}(r)$ :

$$
\omega_{i}(r)=T \frac{\delta \ln Z}{\delta \alpha_{i}(r)} .
$$

To analyze the thermodynamic behavior of the system, one can make a change of variables in the functional integral defining the partition function $Z$. The original meta-Hamiltonian $H\left\{\sigma_{i}(r)\right\}$ depends on a discrete variable $\sigma_{i}(r)$. We use the Hubbard - Stratonovich transformation to re-express the partition function in terms of a continuous lattice variable $\psi$. Let us suppose that the matrix $M_{i j}(r)$ has the form:

$$
M_{i j}(r)=E_{i j} J(r) .
$$

where $E_{i j}$ is a matrix in the local state space, and $J(r)$ describes the dependence of interaction on distance between clusters. Introducing a real, $N$-component "conjugated" field $\psi_{i}(r)$, one rewrites the expression for $Z$ as

$$
\begin{gathered}
Z=\int \prod_{i ; r} D \psi_{i}(r) \exp \left\{-\frac{1}{2 T} \sum_{r, r^{\prime}} J^{-1}\left(r-r^{\prime}\right) E_{i j} \psi_{i}(r) \psi_{j}\left(r^{\prime}\right)\right\} \times \\
\times \prod_{i ; r} \operatorname{Tr} \exp \left[\frac{1}{T}\left(E_{i j} \psi_{i}(r) \sigma_{j}(r)+\alpha_{j}(r) \sigma_{j}(r)\right)\right] .
\end{gathered}
$$

Here, the functional integration $D \psi$ goes over all components $\psi_{i}$ of the field $\psi_{i}(r)$ . The integration path in the complex $\psi$-space may, for a non- positive definite matrix $M_{i j}(r)$, deviate from the real axes to make the integral convergent. The advantage of the form (6) is that now the summation over local states can be easily taken:

$$
\begin{gathered}
Z=\int \prod_{i ; r} D \psi_{i}(r) \exp \left\{-\frac{F}{T}\right\}, \\
F=\frac{1}{2} \sum_{r, r^{\prime}} J^{-1}\left(r-r^{\prime}\right) E_{i j} \psi_{i}(r) \psi_{j}\left(r^{\prime}\right)- \\
-T \sum_{r} \ln \left[\sum_{i} \exp \left\{\frac{1}{T}\left(E_{i j} \psi_{j}(r)+\alpha_{i}(r)\right)\right\}\right] .
\end{gathered}
$$

The new variable $\psi_{i}(r)$ is a continuous one, so one can study the above expression in the saddle-point approximation, in which the only allowed configuration is the saddle point one. In the next approximation one studies then the fluctuations in the vicinity of the saddle point configuration, to find the first fluctuation corrections to the saddle-point approximation. The saddle-point approximation is known to fail at critical points; for phase transitions of the first Ehrenfest order, the saddle-point approximation may be expected to adequately describe the thermodynamics of the system and phase transitions. In the present article we shall restrict ourselves with the first saddle-point approximation.

The saddle-point configuration $\psi(r)$ minimizes the thermodynamic potential $F$ in (7), it obeys the equation:

$$
\begin{gathered}
\sum_{r^{\prime}} J^{-1}\left(r-r^{\prime}\right) \psi_{i}\left(r^{\prime}\right)=Z_{0}^{-1} \exp \left[\frac{1}{T}\left(E_{i j} \psi_{j}(r)+\alpha_{i}\right)\right], \\
Z_{0}=\sum_{i} \exp \left[\frac{1}{T}\left(E_{i j} \psi_{j}(r)+\alpha_{i}\right)\right] .
\end{gathered}
$$


To obtain the generating functional $Z$ one substitutes the solution of this equation in (7). For the probability $\omega_{i}(r)=-\delta F / \delta \alpha_{i}(r)$ one has

$$
\omega_{i}(r)=<\sigma_{i}(r)>=\sum_{r^{\prime}} J^{-1}\left(r-r^{\prime}\right)<\psi_{i}\left(r^{\prime}\right)>.
$$

In the saddle-point approximation, the quantity $F$ in formula (7) plays the role of the nonequilibrium thermodynamic potential [6] 8; at equilibrium, $F$ has a minimum. We define $\omega_{i}(r)$ for nonequilibrium values of $\psi$ by the formula (9). Then, the nonequilibrium potential $F$ may be written in terms of $\omega_{i}(r)$

$$
\begin{gathered}
F=\frac{1}{2} \sum_{r, r^{\prime}} M_{i j}\left(r-r^{\prime}\right) \omega_{i}(r) \omega_{j}\left(r^{\prime}\right)- \\
-T \sum_{r} \ln \left[\sum_{i} \exp \left(\frac{1}{T}\left(\sum_{r^{\prime}} M_{i j}\left(r-r^{\prime}\right) \omega_{j}\left(r^{\prime}\right)+\alpha_{i}\right)\right)\right] .
\end{gathered}
$$

The quantity $\omega_{i}(r)$ may be interpreted as the probability to find the cluster in the $i$-th state. Because of this probabilistic character of the quantity $\omega_{i}(r)$, we will call the saddle point approximation the mean-probability approximation (MPA). The MPA approximation is given by the equations

$$
\frac{\delta F}{\delta \omega_{i}(r)}=0
$$

with an additional condition (3). In the explicit form, this equations may be written as

$$
\begin{gathered}
\omega_{i}(r)=Z^{-1}(r) \exp \left(\frac{E_{i}(r)}{T}\right) \\
E_{i}(r)=\sum_{r^{\prime}} M_{i j}\left(r-r^{\prime}\right) \omega_{j}\left(r^{\prime}\right)+\alpha_{i}(r) \\
Z(r)=\sum_{i=1}^{N} \exp \left(\frac{E_{i}(r)}{T}\right) .
\end{gathered}
$$

Here, $-E_{i}(r)$ may be interpreted as the energy of the cluster when the states of surrounding clusters are characterized by the mean probability $\omega_{i}\left(r^{\prime}\right)$. If the field $\alpha_{i}$ is spatially homogeneous $\left(\alpha_{i}(r)=\alpha_{i}\right)$, the quantities $E_{i}, \omega_{i}$ are, too, spatially homogeneous:

$$
E_{i}=M_{i j} \omega_{j}+\alpha_{i}, \quad M_{i j}=\sum_{r} M_{i j}(r) .
$$

The normalization condition (3) holds for solutions of (12) automatically. The equations (12) have, in general, more than one solution; the number of solutions depends on the values of the parameters $M_{i j}, \alpha_{i}, T$. As mentioned above, the characteristics $M_{i j}, \alpha_{i}$ depend on thermodynamic coordinates of the state (temperature, pressure etc.), and may be used as new thermodynamic variables. Different solutions correspond to different phases of the system. In the vicinity of the first order phase transition, one expects at least two minima of $F$, corresponding to the stable and metastable phases, with the lowest minima corresponding to the stable phase. By specifying the form of the matrix $M_{i j}$, one arrives at different models. Some of these models are discussed in following sections.

\section{Melting of a single-component material, and the Potts model.}

In this section, we discuss a coarsened model for melting in a single-component material [4, 5]. The discussion serves as an introduction to the modeling in the 
next section; a more detailed study of one-component melting may be found in ref. [5]. Here, the material is supposed to preserve the same crystalline local order both in the crystal and in the liquid state. The only degrees of freedom described by the model are the local orientations. To give a coarsened description of orientation order-disorder, one divides the cluster orientation space in angular cells. By definition, all orientations within a given angular cell correspond to the same cluster orientation state, labelled by the angular cell number. The number of cluster orientation states is then determined by the size of an angular cell. With increase of the angular cell size, the description becomes more coarse. We choose this size to model the main features of the system in a most schematic way.

In accordance with the experimental data, the ground state ( the state having the lowest energy) is a crystal, with all clusters occupying the same angular cell. The globally amorphous (but locally crystalline in materials under consideration) global state of the material has a higher energy. This implies an angular attraction between neighboring clusters: the meta-Hamiltonian is at minimum when their orientations coincide and all clusters form an ideal crystal. One could expect that this angular attraction has an angular size, so that the two-cluster contribution to the meta-Hamiltonian increases sharply with the disorientation of the clusters, and then substantially flattens when the mutual disorientation becomes larger than the angular attraction size. We choose the size of the angular cell equal the angular attraction region. Then, a simple schematic way to model the energetics of neighboring cluster interactions is to assign an angular binding energy $J<0$ to two neighboring clusters belonging to the same orientation cell (having the same orientation state). The interaction energy of two neighboring clusters having different orientation states is chosen as the origin of the energy scale $E=0$. The resulting model with only one angular energy characteristics has the form of an $N$ state Potts model with the matrix $M_{i j}$ having the form

$$
M_{i j}=\delta_{i j} J(r),
$$

The assumed equivalence of all orientation states leads to a high symmetry . The parameter $\alpha_{i}$ is now state-independent and may be put equal to zero without loss of generality. In the symmetric high-temperature phase, all $N$ equivalent states have equal probabilities

$$
\omega_{i, h}=\frac{1}{N}, \quad i=1, \ldots, N .
$$

At lower temperatures, this symmetry is spontaneously broken. At these temperatures, one of the states, which we label by $i=1$, is more probable than the others $\left(\omega_{1}>1 / N\right)$, while $\mathrm{N}-1$ states $i=2, \ldots, \mathrm{N}$ have equal probabilities

$$
\left.\omega_{i}\right|_{i \neq 1}=\frac{1-\omega_{1}}{N-1} .
$$


From the minimum condition for the non-equilibrium potential F (see (10)) one obtains the MPA equation (12) (see 4, 5] )

$$
\omega_{1}=Z^{-1} e^{J \nu \omega_{1} / T}, Z=e^{J \nu \omega_{1} / T}+(N-1) e^{J \nu\left(1-\omega_{1}\right) /(N-1) T} .
$$

Here, $\nu J(0)=\sum_{r} J(r)$, with $\nu$ being the effective number of cluster neighbors. Depending on the temperature, the MPA equation has different solutions describing the high-temperature and the low temperature phases. In the high-temperature phase, all states have equal probabilities $\omega_{i, h}=1 / N$, so that the quantity $x=\omega_{1}-\frac{1}{N}$ equals zero. This solution $(x=0)$ formally exists at all temperatures but corresponds to a minimum of $F$, and thus to a stable or metastable state, only at $T>T_{2}$. The low - temperature solution $x \approx(N-1) / N$ exists at $T<T_{1}$. The transition from the low-temperature $(x \neq 0)$ to the high temperature, symmetric phase $(x=0)$ takes place at $T=T_{m}, T_{1}>T_{m}>T_{2}$; this transition is of the first Ehrenfest order (discontinuous). For $N \gg 1$ the temperatures $T_{1}, T_{2}, T_{m}$ may be found in explicit form:

$$
T_{1} \approx \frac{\nu J(0)}{\ln N}, T_{2} \approx \frac{\nu J(0)}{N}, T_{m} \approx \frac{\nu J(0)(N-1)}{2 N \ln N} .
$$

The model may be applied to chemical compositions of stoichiometric compounds behaving like pure materials [9]. The necessary condition of applicability is that the local order should not be destroyed via melting, including the chemical order (the material should not dissociate).

In multicomponent materials there may be a competition of several types of local structure. In a binary system $A-B$, these competing structures may correspond to pure components and to all intermediate stoichiometric compositions. The existence of competing structures enhances the set of local states. In the following section, we generalize the above model to account for two competing types of local order.

\section{System with two competing local structures.}

In this section, we propose and discuss an idealized model of a material with two possible local structures. To account for the type of local structure as a new degree of freedom, we introduce two indices for labelling the local state. The vector $\sigma$ is now written as

$$
\sigma_{i}^{j} ; \quad j=1,2 .
$$

The upper index corresponds to the type of local order, while the lower one numerates the orientations of a cluster. As in the previous section, we assume division of the orientation space into orientation cells, with $n$ orientation states for the first and $m$ for the second local structure. The meta-Hamiltonian of the model may be written in the form

$$
H=-\alpha \sum_{r} \sum_{i=1}^{n} \sigma_{i}^{1}(r)-\sum_{r, r^{\prime}} \sigma_{i}^{k}(r) M_{i l}^{k j}\left(r-r^{\prime}\right) \sigma_{l}^{j}\left(r^{\prime}\right) .
$$


The parameter $\alpha$ describes the internal energy difference between two local structures. As in the case of a one-component system, we choose the angular cell sizes to comprise the angular attraction regions. The kernel $M_{i l}^{k j}(r)$ of interaction is assumed to differ from zero only for nearest neighbors, and to have the form

$$
M_{i l}^{11}(r)=\tilde{J}_{1}(r) \delta_{i l} ; \quad M_{i l}^{22}(r)=\tilde{J}_{2}(r) \delta_{i l} ; \quad M_{i l}^{12}(r)=M_{i l}^{21}(r)=\tilde{\varepsilon}(r)
$$

The interaction between clusters of same composition is chosen in the Potts form discussed in the previous section. The interaction between clusters having different local structures (the term $M^{12}$ and $M^{21}$ ) is assumed to be orientationindependent; the argument here is that if clusters with different structures share a boundary, then the energy of the boundary $\tilde{\varepsilon}$ is mostly due to the difference in the neighboring structures and less due to their relative orientations. Parameters $\tilde{J}_{1}, \tilde{J}_{2}$ characterize then the orientational interaction of same type clusters, and $\tilde{\varepsilon}$ is an inter-structural "surface" energy. We will denote

$$
\varepsilon=\int \tilde{\varepsilon}(r) d V ; \quad J_{1}=\int J_{1}(r) d V ; \quad J_{2}=\int \tilde{J}_{2}(r) d V .
$$

¿From symmetry arguments, one has for the mean values of the parameter $\omega$ (see the previous section):

$$
\begin{gathered}
\omega_{1}^{1}=\omega_{1} ; \quad \omega_{k \neq 1}^{1}=\frac{x-\omega_{1}}{n-1} \\
\omega_{1}^{2}=\omega_{2} ; \quad \omega_{k \neq 1}^{2}=\frac{1-x-\omega_{2}}{m-1} .
\end{gathered}
$$

Here, $x$ is the concentration of clusters having the first structure. The quantities $\omega_{1}$ and $\omega_{2}$ in (23) describe the orientational ordering; the lower index 1 labels the preferred orientation in the orientationally ordered (crystalline) state. Now one is able to classify the possible phases in the model:

1. $\omega_{1} \sim x, x \sim 1$ - crystal with the first type of local structure (C1);

2. $\omega_{1}=x / n, x \sim 1$ - liquid with the first type of local structure (L1);

3. $\omega_{2} \sim 1-x, x \sim 0$ - crystal with the second type of local structure (C2);

4. $\omega_{2}=(1-x) / m, x \sim 0$ - liquid with second type of local structure (L2)

The nonequilibrium thermodynamic potential $F$ defined by formula (10) may be calculated using the meta-Hamiltonian of the model; some lengthy algebra then leads to the form

$$
\begin{gathered}
F=\frac{1}{2}\left(J_{1} \omega_{1}^{2}+J_{1} \frac{\left(x-\omega_{1}\right)^{2}}{n-1}+J_{2} \omega_{2}^{2}+J_{2} \frac{\left(1-x-\omega_{2}\right)^{2}}{m-1}\right)+ \\
+\varepsilon x(1-x)-T \ln \left\{\exp \left(\frac{J_{1}}{T} \omega_{1}+\frac{\varepsilon}{T}(1-x)+\frac{\alpha}{T}\right)+\right. \\
\quad+(n-1) \exp \left(\frac{J_{1}\left(x-\omega_{1}\right)}{T(n-1)}+\frac{\varepsilon}{T}(1-x)+\frac{\alpha}{T}\right)+ \\
\left.+\exp \left(\frac{J_{2}}{T} \omega_{2}+\frac{\varepsilon}{T} x\right)+(m-1) \exp \left(\frac{J_{2}\left(1-x-\omega_{2}\right)}{T(m-1)}+\frac{\varepsilon}{T} x\right)\right\}
\end{gathered}
$$

The minima of this function determine equilibrium phases and the phase diagram of the model; we study the phase boundaries in the following section. 


\section{Binary system with limited miscibility.}

Consider a binary system in which the two local structures correspond to phases with predominance of component $A$ and $B$ respectively. If these phases are stoichiometric compositions, the model describes the material in some range of intermediate concentrations; we assume that no other stoichiometric composition can add a third type of local order. We consider systems in which the two types of local order differ sharply (have different groups of local symmetry or incompatible interatomic distances). Systems with limited miscibility usually exhibit a sharp decrease of melting temperature with deviation from the stoichiometric compound 9, 10]. The experimentally investigated phase equilibria and the phase diagrams in these materials may be classified as follows [12, 13]:

1. Systems with limited miscibility both in the liquid and solid state (the diagram with eutectic and monotectic equilibria), for example $\mathrm{Pb}-\mathrm{Zn}, \mathrm{Cu}-$ $P b, G a-P b$.

2. Systems with limited miscibility in the solid state and unlimited miscibility in the liquid one (the diagram with single eutectic equilibrium), for example $\mathrm{Ni}-\mathrm{Cr}, \mathrm{Cu}-\mathrm{Ag}, \mathrm{Cu}-\mathrm{CuMg}$.

The concentration of the second component determines the volume parts $x$ and $1-x$ of clusters having the 1 and the 2 structures respectively. Let us study the phase diagram of the model in the $(x, T)$ plane (Fig.1).

The MPA equations (12) may be written as

$$
\begin{gathered}
\tilde{\omega}_{1}=\frac{\omega_{1}}{x}=\left\{1+(n-1) \exp \left(\frac{J_{1}}{T} x \tilde{\omega}_{1} \frac{1-n \tilde{\omega}_{2}}{n-1}\right)\right\}^{-1} \\
\tilde{\omega}_{2}=\frac{\omega_{2}}{1-x}=\left\{1+(m-1) \exp \left(\frac{J_{2}}{T}(1-x) \frac{1-m \tilde{\omega}_{2}}{m-1}\right)\right\}^{-1} \\
x=\left\{1+\frac{\tilde{\omega}_{1}}{\tilde{\omega}_{2}} \exp \left(\frac{J_{2}}{T}(1-x) \tilde{\omega}_{2}-\frac{J_{1}}{T} x \tilde{\omega}_{1}-\frac{\varepsilon}{T}(1-2 x)-\frac{\alpha}{T}\right)\right\}^{-1} .
\end{gathered}
$$

When $x=0$ or $x=1$, these equations coincide with those of the Potts model for the melting of pure components. The apparent effect of the second component is the lowering of the melting temperature. Besides that, the redistribution of components between liquid and solid phases [6] results in two-phase domains $(L 2, C 2)$ and $(L 1, C 1)$ which are restricted by the lines $x_{3}(T), x_{4}(T)$ and $x_{5}(T), x_{6}(T)$ respectively (see Fig.1). The boundaries of a two-phase domain may be found by standard procedure from equilibrium free energies of both coexisting phases; to find the equilibrium free energy $F_{e q}(x, T)$, one applies the method of Lagrange multipliers:

$$
\begin{aligned}
-F_{e q}(x, T) & =J_{2}(1-x)^{2}\left(\widetilde{\omega}_{2}-\frac{\widetilde{\omega}_{2}^{2}}{2}-\frac{\left(1-\widetilde{\omega}_{2}\right)^{2}}{2(m-1)}\right)+J_{1} x^{2}\left(\widetilde{\omega}_{1}-\frac{\widetilde{\omega}_{1}^{2}}{2}-\frac{\left(1-\widetilde{\omega}_{1}\right)^{2}}{2(n-1)}\right)+ \\
& +\varepsilon x(1-x)-T(1-x) \ln \left[\widetilde{\omega}_{2}(1-x)\right]-T x \ln \left[\widetilde{\omega}_{1} x\right] .
\end{aligned}
$$

The phase equilibrium conditions for, e.g., the domain $(L 2, C 2)$ are

$$
\begin{aligned}
F_{e q, C 2}\left(T, x_{3}\right)-\frac{\partial F_{e q, C 2}\left(x_{3}, T\right)}{\partial x_{3}} x_{3} & =F_{e q, L 2}\left(T, x_{4}\right)-\frac{\partial F_{e q, L 2}\left(x_{4}, T\right)}{\partial x_{4}} x_{4} ; \\
\frac{\partial F_{e q, C 2}\left(x_{3}, T\right)}{\partial x_{3}} & =\frac{\partial F_{e q, L 2}\left(x_{4}, T\right)}{\partial x_{4}} .
\end{aligned}
$$


Here, the indexes $C 2$ and $L 2$ refer to the phase. For the case $m, n \gg 1$, these conditions may be obtained in explicit form:

$$
\begin{gathered}
\frac{J_{2}}{2}\left(1-x_{3}^{2}\right)+\varepsilon x_{3}^{2}-\frac{J_{1}}{2 n} x_{3}^{2}-T \ln \left(1-x_{3}\right)= \\
=\frac{J_{2}}{2 m}\left(1-x_{4}^{2}\right)+\varepsilon x_{4}^{2}-\frac{J_{1}}{2 n} x_{4}^{2}-T \ln \left(1-x_{4}\right)+T \ln m ; \\
T \ln \frac{x_{3}}{1-x_{3}}+J_{2}\left(1-x_{3}\right)+2 x_{3} \varepsilon-\frac{J_{1}}{n} x_{3}= \\
=T \ln \frac{m x_{4}}{1-x_{4}}+\frac{J_{2}}{m}\left(1-x_{4}\right)+2 x_{4} \varepsilon-\frac{J_{1}}{n} x_{4}
\end{gathered}
$$

At $x=0$ or $x=1$ these relations give (18) for the melting temperature of pure material. For a small miscibility $(x \ll 1)$ one has approximately

$$
\frac{\partial x_{3}}{\partial T}=k_{3}, \quad \frac{\partial x_{4}}{\partial T}=k_{4},
$$

with $(m, n \gg 1)$ :

$$
k_{3}=-\frac{2 \ln m}{T_{2}+J_{1} / n-2 \varepsilon}, \quad k_{4}=m k_{3} .
$$

The conditions determining $x_{5}(T)$ and $x_{6}(T)$ may be found in the same way.

The miscibility gaps both in liquid and solid states and the occurrence of two-phase domains $(L 1, L 2)$ and $(C 1, C 2)$ are due to the "interface tension" $\varepsilon$ between two structures. The width of the domain $(L 1, L 2)$ is limited from above by the gap (fig.2). From the above equations one obtains the relations between the parameters of the gap $T, x, x^{*}$ (see fig.2) as

$$
\begin{gathered}
F(x)=F\left(x^{*}\right), \\
F(x)=-\frac{J_{2}}{2 m} x^{2}+\frac{J_{1}}{2 n} x^{2}+\varepsilon x^{2}-T \ln (1-x) ; \\
T \ln \frac{(1-x) x^{*}}{\left(1-x^{*}\right) x}=\left(\frac{J_{2}}{m}+\frac{J_{1}}{n}-2 \varepsilon\right)\left(x^{*}-x\right) .
\end{gathered}
$$

Above the domain $(L 1, L 2)$ in the phase plane the system is homogeneous. The line separating this domain from the homogeneous phase is a line of phase transitions. The phase transition is of the first Ehrenfest order anywhere except the top of the gap with coordinates $x_{c}, T_{c}$ in the $x-T$ plane

$$
x_{c}=\frac{1}{2} ; \quad T_{c}=\frac{J_{2}}{4 m}+\frac{J_{1}}{4 n}-\frac{\varepsilon}{2},
$$

where it is of second order. The two-phase domain $(C 1, C 2)$ is limited by lines $x_{1}, x_{2}$ (see fig.3). At low temperatures, or for large $m, n \gg 1$ one may approximate the equations as

$$
\begin{aligned}
& \frac{J_{2}}{2}\left(1-x_{1}^{2}\right)-\frac{J_{1}}{2 n} x_{1}^{2}+\varepsilon x_{1}^{2}-T \ln \left(1-x_{1}\right)= \\
& =-\frac{J_{1}}{2} x_{2}^{2}+\frac{J_{2}}{2 m}\left(1-x_{2}^{2}\right)+\varepsilon x_{2}^{2}-T \ln \frac{1-x_{2}}{m} ; \\
& J_{2}\left(1-x_{1}\right)-\frac{J_{1}}{n} x_{1}+2 \varepsilon x_{1}-T \ln \frac{n\left(1-x_{1}\right)}{x_{1}}= \\
& =\frac{J_{2}}{m}\left(1-x_{2}\right)-J_{1} x_{2}+2 \varepsilon x_{2}-T \ln \frac{1-x_{2}}{m x_{2}} .
\end{aligned}
$$

Continuing all lines obtained until their crossing points and using the Gibbs phase rule, one gets the full phase diagram of the system. Fig.4 schematically 
shows the phase diagram of a system with monotectic and eutectic equilibriums. If the crossing point of lines $x_{4}, x_{6}$ lies in the homogeneous phase, the phase diagram degenerates into that with single eutectic equilibrium (see Fig.5). The values of $x_{a}, x_{b}, x^{*}, T^{*}$ (see fig.5) are related by

$$
\begin{gathered}
F_{e q, C 2}\left(x_{a}, T^{*}\right)-\frac{\partial F_{e q, C 2}\left(x_{a}, T^{*}\right)}{\partial x_{a}} x_{a}= \\
=F_{e q, L}\left(x^{*}, T^{*}\right)-\frac{\partial F_{e q, L}\left(x^{*}, T^{*}\right)}{\partial x^{*}\left(x_{b}, T^{*}\right)} x^{*} \\
=F_{e q, C 1}\left(x_{b}, T^{*}\right)-\frac{\partial F_{e q, C 1}\left(x_{b}\right.}{\partial x_{b}} x_{e q, C 1}\left(x_{b}, T^{*}\right) \\
\frac{\partial F_{e q, C 2}\left(x_{a}, T\right)}{\partial x_{a}}=\frac{\partial F_{e q, L}\left(x^{*}, T^{*}\right)}{\partial x^{*}}=\frac{\partial F^{*}}{}
\end{gathered}
$$

where the thermodynamic potentials $F_{e q}$ for corresponding phases are defined in (26).

\section{Polymorphous Materials}

The model may be also used, with minor changes, to describe polymorphic transitions in a single-component material. In this case two different local structures are the competing local arrangements of the same atoms, so the parameter $x$ is no longer conserved. The thermodynamic potential (24) and the MPA approximation (25) give the phase diagram in the $(\alpha, T)$ thermodynamic plane (Fig.6). Starting from one of the phases one changes the parameter $\alpha$ (the difference of local energies of the two arrangements playing the role of a thermodynamic coordinate of the state), and arrives on a polymorphic phase transition $C 2 \rightarrow C 1$ at $\alpha \approx J_{2}-J_{1}$ in the solid state. This line may have a continuation in the liquid area, where it terminates in the critical point:

$$
\begin{gathered}
\alpha_{c}=\frac{J_{2}}{m}-\varepsilon+T_{c} \ln \frac{m}{n}-2 T_{c} \\
T_{c}=\frac{1}{4}\left[\frac{J_{1}}{n}+\frac{J_{2}}{m}-2 \varepsilon\right]
\end{gathered}
$$

Below $T_{c}$, the local orders are well distinguished, and phases $L 1$ and $L 2$ are divided by the line of the first order phase transitions with a critical point at the end of the line (see the line $\mathrm{AB}$ in Fig.6). The polymorphic liquid-liquid phase transitions were predicted and theoretically studied earlier 11]; recently, this prediction was confirmed by experiments. 22, 19] The discovery of liquidliquid phase transitions in single-component materials gives a strong argument in favor of a local order in at least some melts.

\section{Discussion of the theory}

The model proposed in this paper is rather schematic, it operates with only a few parameters characterizing the material, and does not pretend to give a precise quantitative description. At the same time, it gives a qualitatively correct description of a surprisingly wide range of known phenomena, and in particular predicts the topology of the binary system phase diagram. The proposed model may be compared with other known models for the melting transition. 
An early model of melting is the Landau theory with a scalar order parameter ([6], see also [14]) in which a cubic term is postulated to result in a first order phase transition. In this approach, the nature of the transition and the shortrange order in the material are not considered. On the opposite extreme, one can calculate the thermodynamic potentials for the liquid and crystalline phases using "realistic" interatomic interaction potentials [15, by numerical techniques. Our approach is intermediate. The local order in the material has to be found from molecular-scale calculations (or from experiment). This local order is supposed to be a feature both of the crystalline phase and of the melt. The possibility of crystalline local order in the melt is not expected to be justified for all liquids; the conditions for such a feature is discussed in [5]. Both strong covalency and local potentials of three (or more) body type (steric interactions, hydrogen bonding, ...) favor the local crystalline local order in the melt.. For materials with crystalline local order both in the solid and in the molten states, the phases may be described in terms of the local structure, using the orientation order parameter. This also allows one to address the problem of polymorphic phase transitions in the melt (see sec.4.2).

A special situation appears when the competing structures have substantially different densities. The change $\alpha$ of the internal energy upon changing the type of the local structure then includes the large work against the external pressure. In such materials, melting may coincide with a polymorphic transition. This may explain the "anomalous" melting of covalent crystals with diamond structure $(C, S i, G e)$ and compositions of the type $A_{I I I} B_{V}(\operatorname{In} S b, G a S b)$ with sphalerite structure. The melting of these substances at modest pressures is accompanied by a change of the local order, and the coordination number has nearly $100 \%$ increase 16, 17. The large density increase causes the semiconductor - metal transition during melting [16. At high pressures, these substances transform into metallic crystals with the structure of white $S n$ [18. One expects that there exists a line of the semiconductor - metal transition which crosses the melting line of crystal melting. At this point there is a three - phase equilibrium of the covalent crystal, the metallic crystal, and the metallic liquid corresponding to the point $A$ on fig.4. In principle, the coexistence line of the crystal and liquid having different local structures may terminate (at a lower pressure) at the crossing point of the melting line and the line of metallic liquid - covalent liquid transition. This line of polymorphic liquid -liquid transition extends then into the liquid area and terminates in the liquid -liquid critical point. Recently, a liquid-liquid phase transition line of that sort was discovered in selenium [19. A liquid-liquid phase transition with loss of the metallic conductivity was long ago predicted by Landau and Zel'dovich 20].

The "covalent" melting takes place at high temperatures [21. At lower temperatures, polymorphic phase transitions in the melt may be expected in materials having low-coordination covalent modifications (with the coordination number less than 4). Melting of such materials does not require large temperatures because the energetics is determined by the non-covalent bonds. Then, at high pressures, the covalent melt must transform into a metal one. Recently, liquid-liquid transitions were found in liquid $T e, B i$ and $S e$ 22]. 
Qualitatively, the phase diagram for Se has the shape shown in Fig.6. The many liquid-liquid phase transitions in Te and Be evidence that there are several competing local structures in the single component liquids. Compositions of the type $A_{I V} B_{V I},\left(A_{V}\right)_{2}\left(B_{V I}\right)_{3}$ with covalent melting [16] (GeTe, SnTe .f $\mathrm{PbTe}, \mathrm{PbSe}, \mathrm{PbS}, \mathrm{Bi}_{2} \mathrm{Se}_{3}, \mathrm{Bi}_{2} \mathrm{Te}_{3}, \mathrm{Sb}_{2} \mathrm{Te}_{3}$ ), may be candidates for liquid-liquid metal-covalent transitions. If one supposes that at high pressure any substance turns out to be a metal [20], then this phase transition should take place for the elements of V and VI groups, but it is unclear whether it is accompanied by a polymorphic transition. The local state representation allows one to model the changes of the global order in condensed materials having a "hard" local order. This order may have components other than the structure (the local packing of atoms, and the orientation of the local structural anisotropy). Similar models are being used to describe magnetic 23, 24 and ferroelectric 25] ordering. These general behaviors can, as we have shown, be understood in terms of a very simple Potts-type model that includes only a small number of physical parameters characterizing the energy difference between local crystalline states, and the energy due to interfacial interaction between two neighboring clusters of same or different types. These parameters are physically meaningful, and they seem to be adequate to discuss the phase diagram of binary systems.

\section{Acknowledgments}

We thank Boris I. Shumilo, Antoni C.Mitus and Michael V.Chertkov for many discussions of crystalline ordering. This work was supported by NASA (Grant NAG3-1932), the Chemistry Division of the ONR, and by the CAMP collaborative MVRI program of the ONR and by the NSF-DMR through the Northwestern University MRC (Grant DMR 9120521). 


\section{References}

[1] R.E.Reed-Hill,Physical Metallurgy Principles, PWS-KENT Publishing Comp., Boston, 1973.

[2] J.-P.Hansen, I.R. McDonald. The theory of Simple Liquids, 2-nd edition, Academic Press, London 1990..

[3] A.R.Ubbelohde. The Molten State of Matter. Wiley, New York, 1978.

[4] A.C.Mitus, A.Z.Patashinski, Sov.Phys. JETP, 53, 798 (1981); Phys. Lett. 87A, 179 (1982).

[5] A.Z.Patashinski, M.A.Ratner. Inherent amorphous structures and statistical mechanics of melting, Submitted to J.Chem. Phys. (May 1996)

[6] L.D.Landau, E.M.Lifshitz. Statistical Physics, Part 1, Pergamon, Oxford 1986

[7] A.Z.Patashinski, M.V.Chertkov. Local State Representation in Statistical Mechanics of Condensed matter. Preprint INP 91-51, Novosibirsk 1991.

[8] A.Z.Patashinski, V.L.Pokrovski. Fluctuation theory of phase transitions. Pergamon, Oxford, 1979.

[9] Intermetallic Compounds. Edited by J.H.Westbrook, John Wiley \& Sons, Inc., New York - London - Sydney, 1975.

[10] H.J.Goldsmith. Interstitial Alloys. Butter Vorth, London, 1967.

[11] A.C.Mitus, A.Z.Patashinski, B.I.Shumilo,Phys. Lett. 113A,41 (1985); A.C.Mitus, A.Z.Patashinski. Acta Phys. Pol., 38, 10105 (1988).

[12] R.W.Cahn, P.Hansen. Physical Metallurgy. North - Holland, Amsterdam Oxford - New York - Tokyo, 1983

[13] M.Hansen. Constitution of binary alloys. Mc-Graw Hill, New York, 1958.

[14] T.Kitamura, Physica A, 160, 181 (1989).

[15] H.O.Carmesin, Phys. Rev. B, 41, 4349 (1990).

[16] V.M.Glazov, S.N.Chizhevskaya, N.N.Glagoleva, Zhidkie poluprovodniki. Nauka, Moscow 1967 (in Russian);

[17] A.F.Ioffe, A.R.Regel. Progr. Semicond. 4, 237 (1960).

[18] G.H. Drickamer. in: Phase stability in metals and alloys, ed. by P.S.Rudman, Mc-Graw Hill, New York, 1967

[19] V.V.Brazhkin, R.N.Voloshin, S.V.Popova, A.G.Umnov, Phys. Lett.A 154, $413(1991)$ 


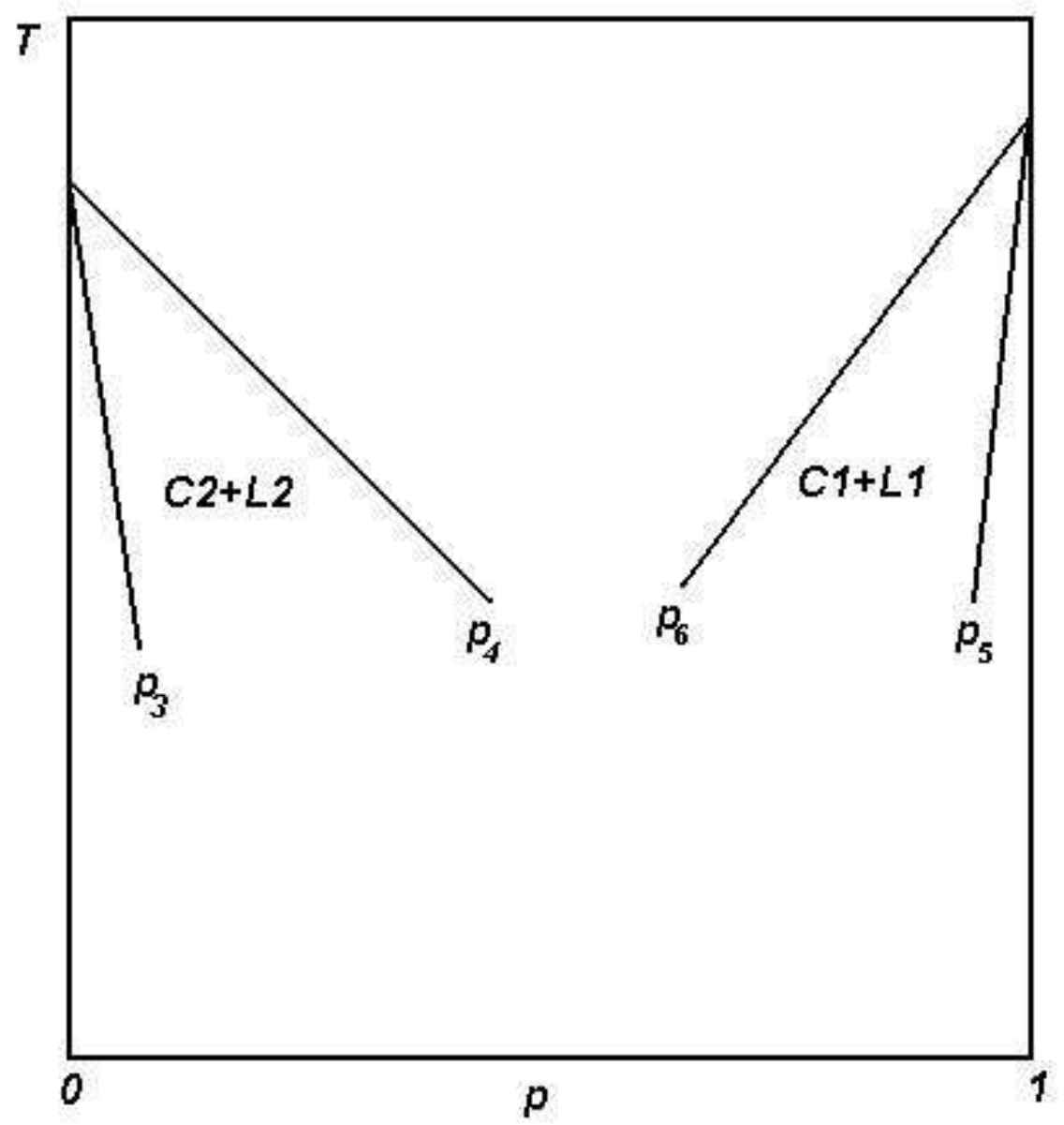

Figure 1: The thermodynamic $(x-T)$ plane of a binary system. The two phase domains of liquid and crystal coexistence are drawn. 


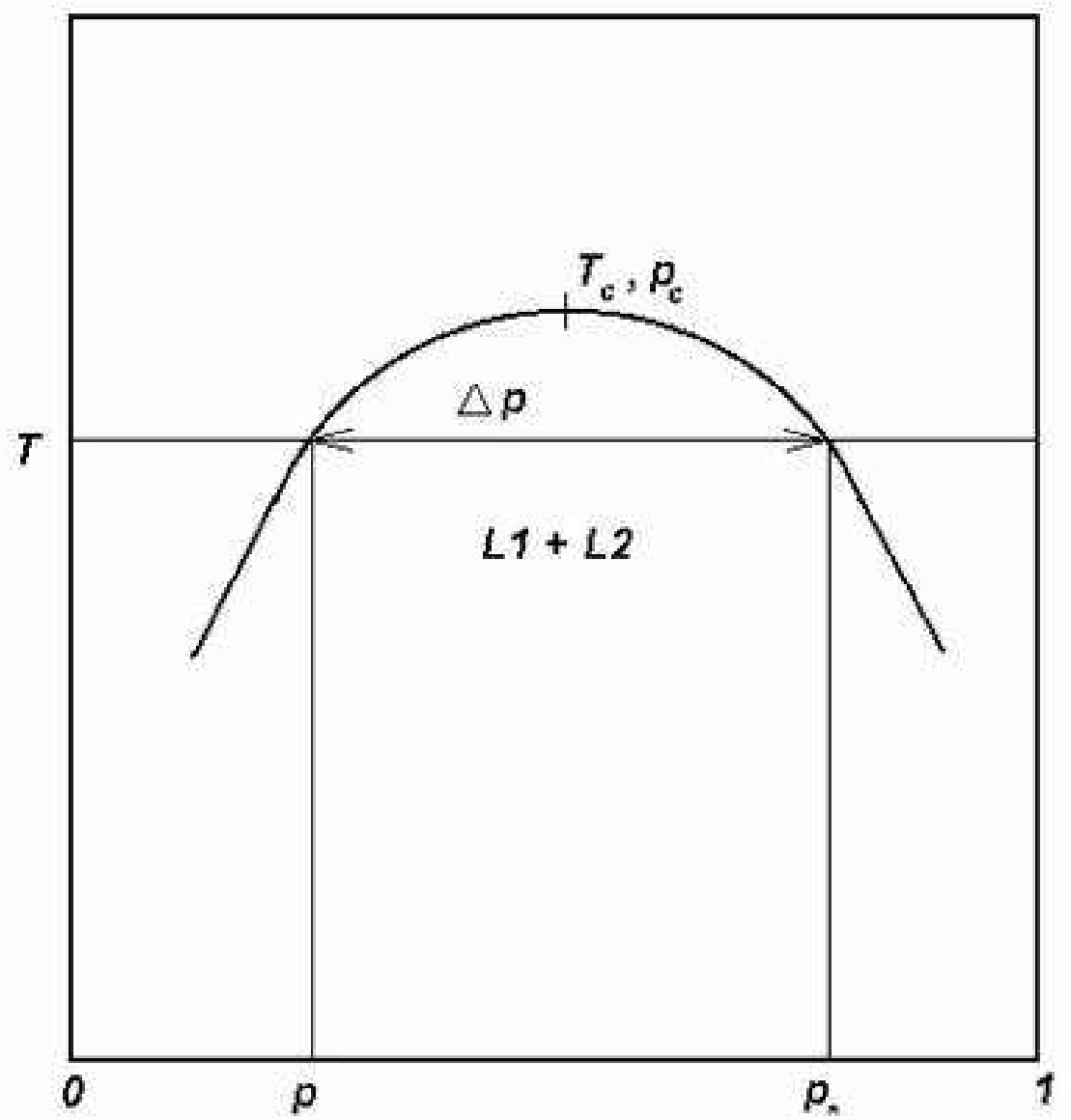

Figure 2: The miscibility gap in the liquid state. The position of the gap corresponds to eqn. (31). 


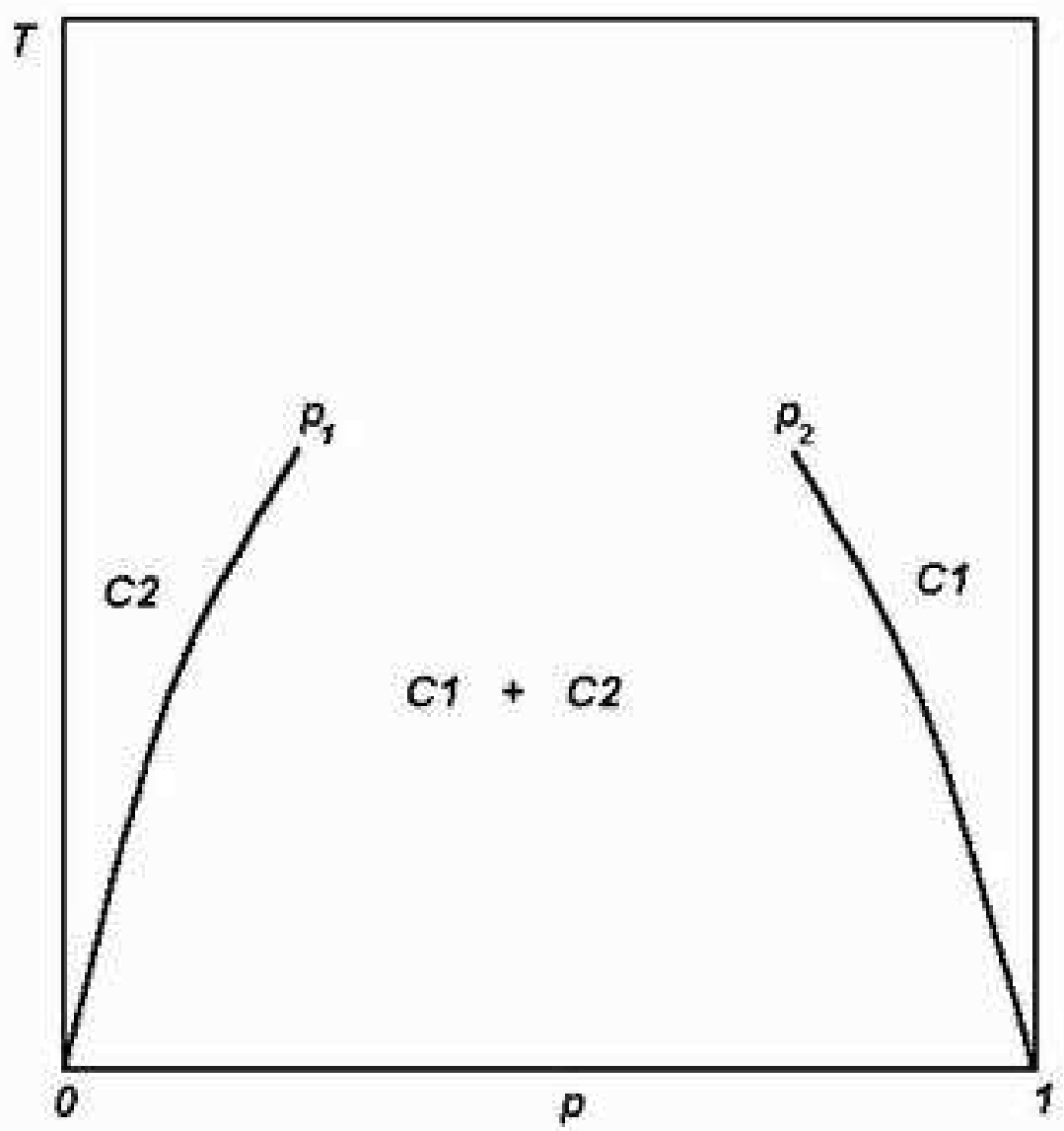

Figure 3: The two-phase domain $\left(\mathrm{C}_{1}\right.$ and $\left.\mathrm{C}_{2}\right)$ in the thermodynamic plane of the system. 


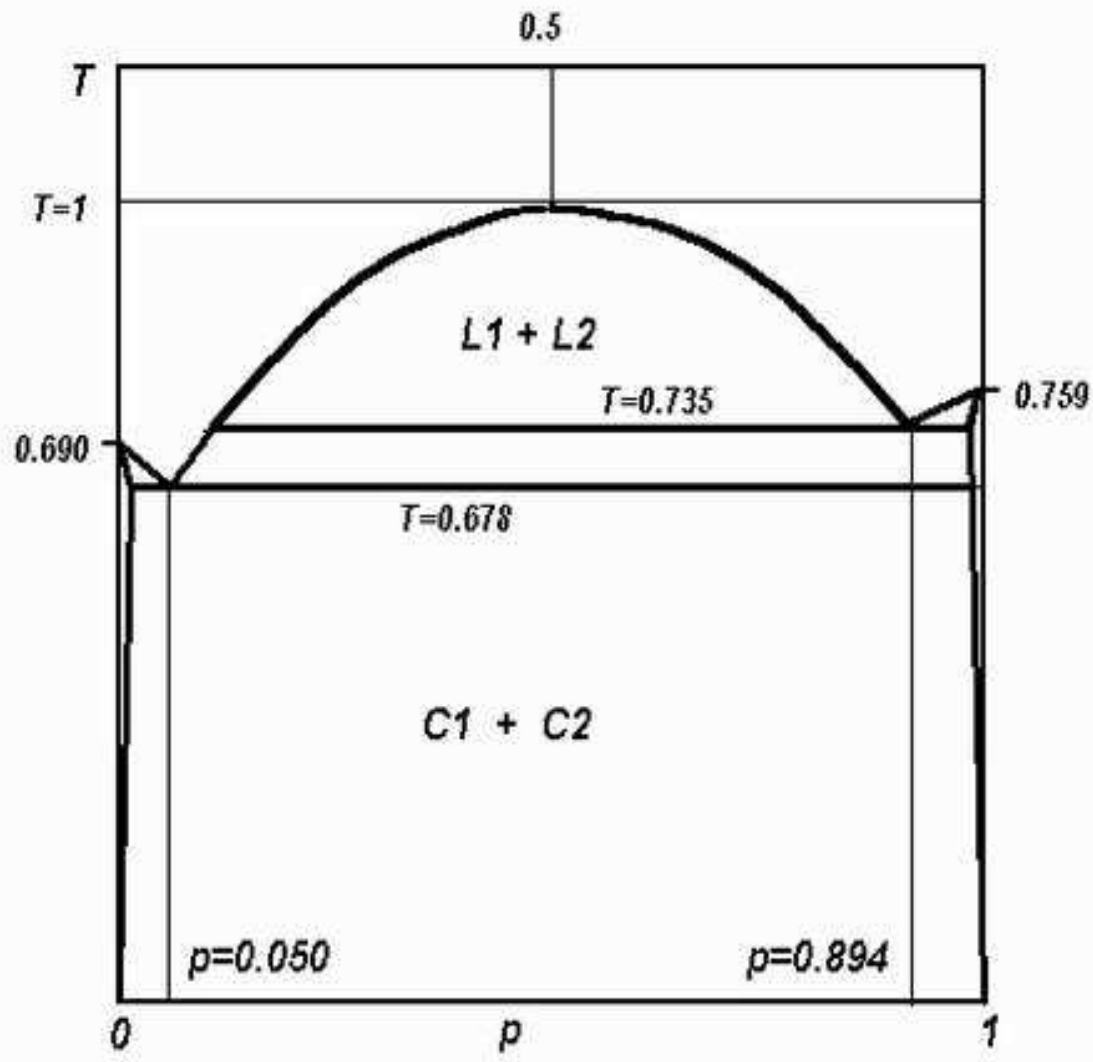

Figure 4: The phase diagram of a system with monotectic and eutectic equilibrium. 


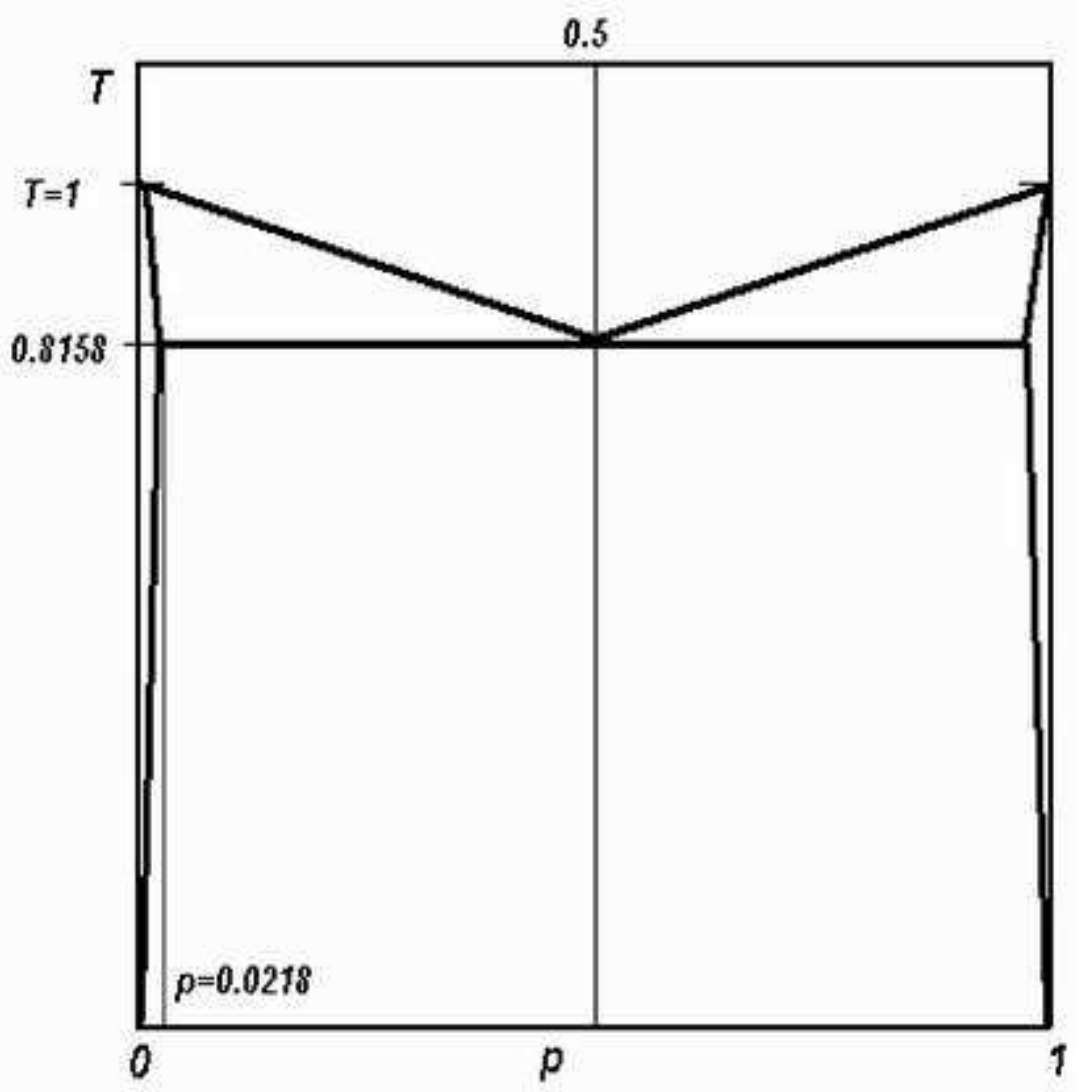

Figure 5: The phase diagram of a system with a single eutectic equilibrium. 


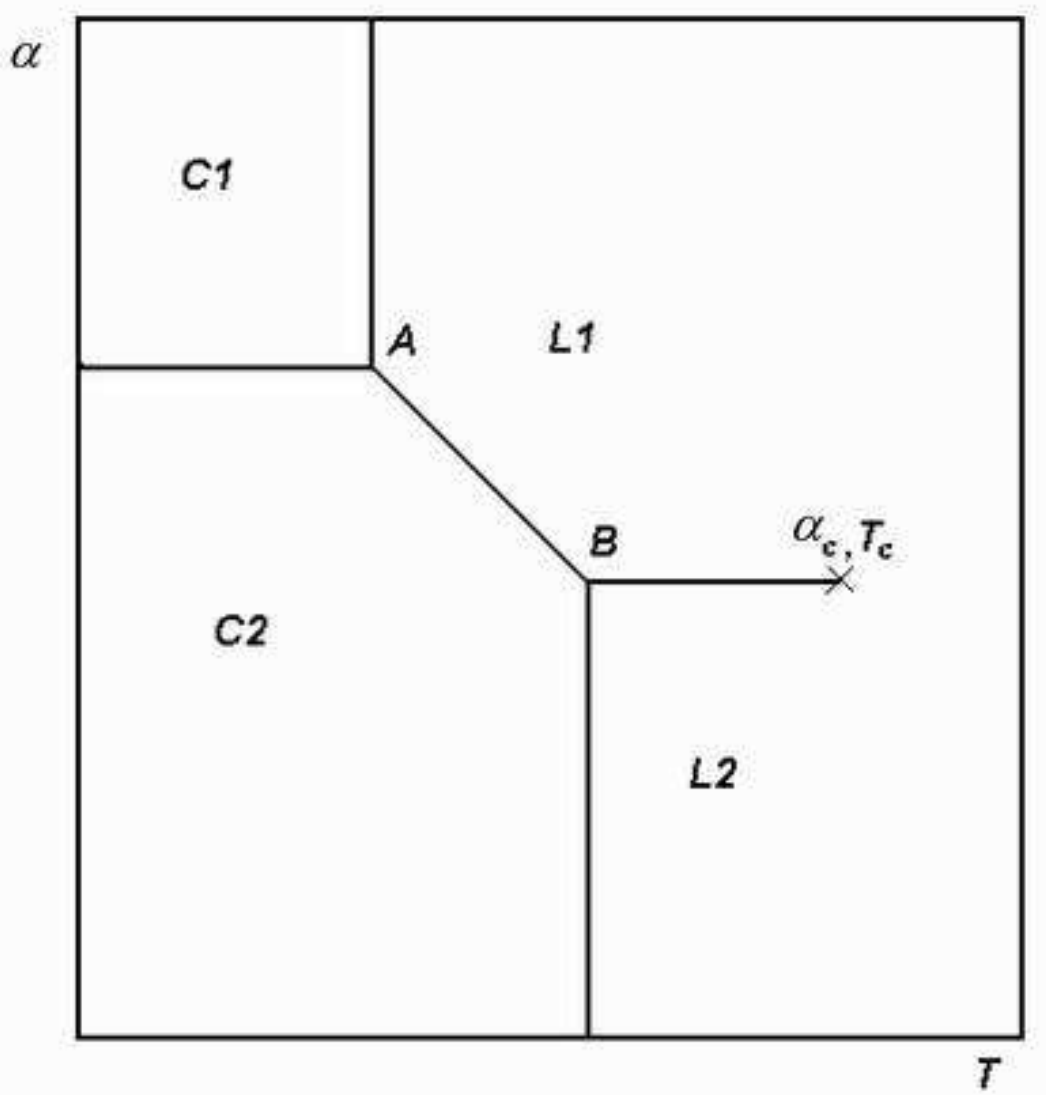

Figure 6: The phase diagram of a polymorphous system. 
[20] L.D.Landau, Ya.B.Zel'dovich, Zh. Eksp. Teor. Fiz. (Sov. Phys. JETP) 14, $32(1944)$

[21] N.F.Mott, E.A.Davis. Electron Processes in Non - Crystalline Materials, Clarendon Press, Oxford, 1979.

[22] V.V.Brazhkin, R.N.Voloshin, S.V.Popova, A.G.Umnov, J.Phys.C., 4, 1419; 1427 (1992); JETP Lett. 50, 424 (1989).

[23] A.F.Andreev, V.I.Marchenko. Zh.Eksp.Teor.Fiz., 70, 1522 (1976)

[24] S.V.Vonsovsky. Magnetism. Nauka, Moscow, 1971 (in Russian).

[25] D.Jona, F.Shirane. Ferroelectric Crystals. Pergamon Press, Oxford 1962. 